\title{
Low-cost equipment for schedule generation III: Variable ratio and ancillary equipment
}

\author{
L. J. AYLESBURY and R. GLYNN OWENS \\ University of Oxford, Oxford, England $O X 37 J X$
}

\begin{abstract}
Circuits are described that extend earlier designs for schedule-generating equipment using complementary metal oxide semiconductor (CMOS) logic. Specifically, the circuits consist of a variable-ratio generator and a series of circuits providing ancillary equipment, including a simple elapsed-timer meter, a logic probe, a variety of logic functions, a pulse generator, and circuits for interfacing. All circuits are compatible with those previously described.
\end{abstract}

Earlier papers (Aylesbury \& Owens, 1977; Owens \& Aylesbury, 1978) described a variety of low-cost circuits for the generation of reinforcement schedules. An important schedule not covered by these papers is the variable-ratio (VR) schedule (Ferster \& Skinner, 1957). In this, a certain number of responses must be emitted by the organism to obtain reinforcement, this number varying on each occasion. The present paper describes such a circuit, together with various ancillary circuits having a variety of uses in conjunction with the equipment already described. The ancillary circuits include a logic panel, supplying simple AND, OR, inverter, and flip-flop functions, enabling several circuits to be combined in order to produce more complex schedules of reinforcement. Also described are a circuit providing a clearing of all units when equipment is first switched on, and two extra pieces of equipment of use in setting up and running the equipment, a logic probe and a simple elapsed-time meter. The most complex circuit is, however, that producing a VR schedule, and this will be discussed first.

\section{THE VARIABLE-RATIO GENERATOR}

The VR generator provides a schedule whereby a sequence of 16 different ratios may be programmed in advance of, or even during, an experiment. By means of a nickel-cadmium battery backup, a small current is supplied to the necessary integrated circuits (ICs) when the equipment as a whole is switched off, enabling a sequence of ratios to be retained in the memory if desired.

The logical structure of this circuit is shown in Figure 1. The basis of the circuit is a set of three ICs each providing 16 4-bit memory locations, here combined as 16 12-bit addresses. The addresses contain the numbers used in the

R. Glynn Owens is now Senior Psychologist, H.M. Prison, Camp Hill. This work was completed while the second author was in receipt of a grant from the Medical Research Council of Great Britain, to whom thanks are due. Reprints may be obtained from L. J. Aylesbury, University of Oxford, Department of Psychiatry, Warneford Hospital, Oxford, England OX3 7JX. ratio sequence and are read sequentially during the course of an experiment. That is, the device can store 16 sequences of 16 ratios each, the individual ratios being represented by 12 -bit binary numbers that allow ratios of from 1 to 4,095. Input events (pulses, for example, from a microswitch operation) are taken through a special interface (described below) to the binary counter IC 1. The number of pulses detected is represented as outputs Q1-Q12 of IC 1; these outputs are compared with the word currently in the memory formed by ICs 3-5 using exclusive-NOR Gates 6-8a-d. (The truth table for an exclusive-NOR gate is given in Figure 1.) When the two numbers (in memory and in IC 1) correspond, all outputs of the NOR gates are high, which causes (via the AND gates formed by ICs $9,11 \mathrm{a}$, and 10a) the output of IC $10 \mathrm{~b}$ to go high, triggering the monostable, which, through appropriate interfacing (below), provides the VR output pulse. The pulse from the monostable is also taken to the clock input of IC 2 (the memory address selector), causing this to increment and thus select the next word in the stored sequence. IC 2 is a binary counter, the outputs of which are connected to the address inputs of the memory ICs 3-5. The same pulse that increments IC 2 also resets IC 1 ready for the next count (via OR Gate 12A).

The memory ICs each contain a total of 256 locations. In the circuit shown in Figure 1, provision is made for these locations to store up to 16 different sequences. Selection of a particular one of these sequences is made using a hexadecimal $(0-9, \mathrm{~A}-\mathrm{F})$ thumbwheel switch, $\mathrm{S} 1$, connected to the remaining address lines of ICs 3,4 , and 5 . The position in a sequence that has been reached at any particular moment is given by LED 1 , a hexadecimal display connected to the address lines via buffers IC 13 . Figure 2 gives the detailed wiring diagram for the circuit. The memory devices are low-cost units made by INTEL. These and the hexadecimal display require 5-V supplies rather than the $12 \mathrm{~V}$ used in the remainder of the system. A separate power supply is therefore needed for this circuit; a suitable design is shown in Figure 3. The power supply incorporates three $1.25 \mathrm{~V}$ nickelcadmium batteries, which provide $3.75 \mathrm{~V}$ to the $\mathrm{Vcc}$ 


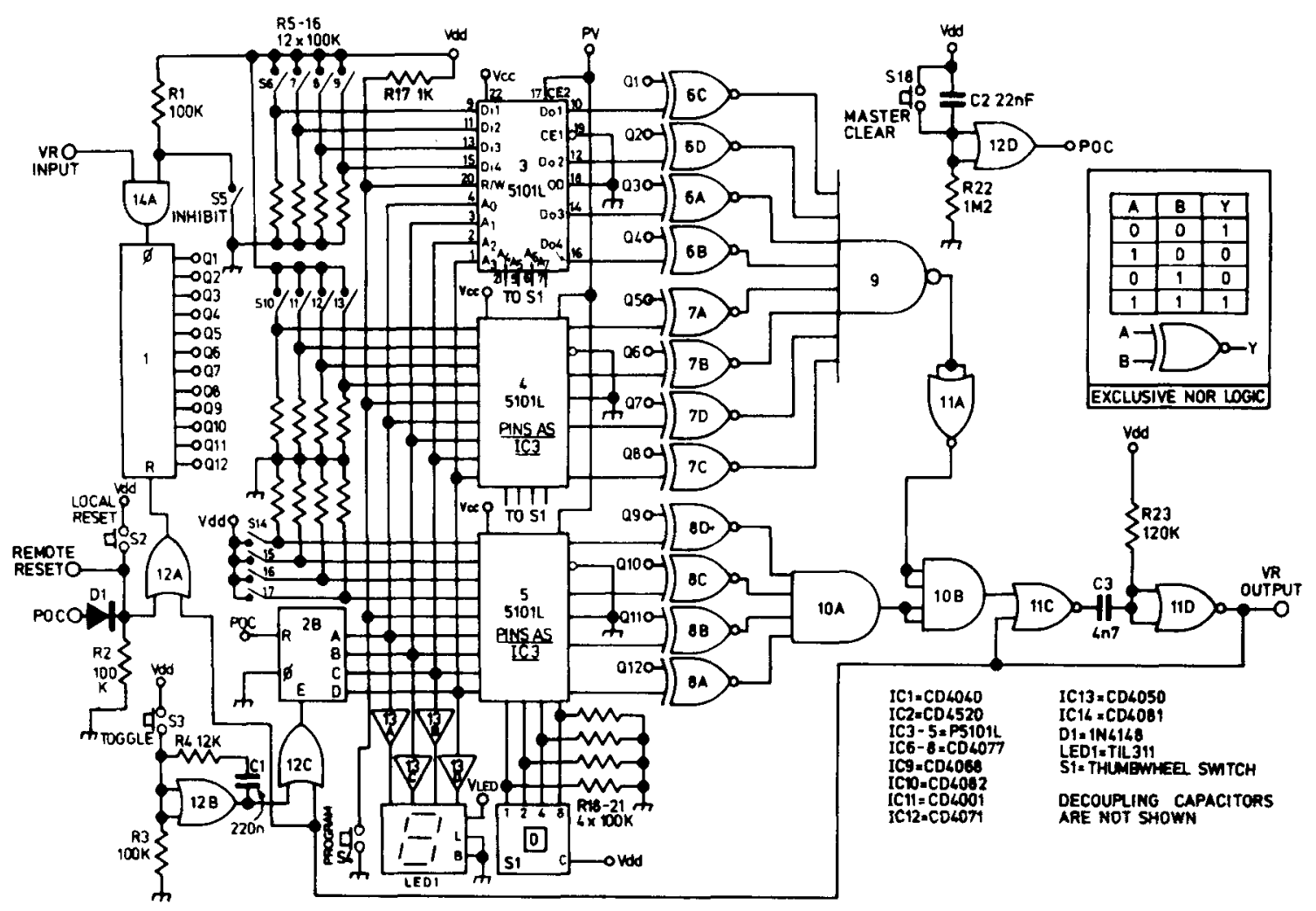

Figure 1. Logical structure of VR circuit.

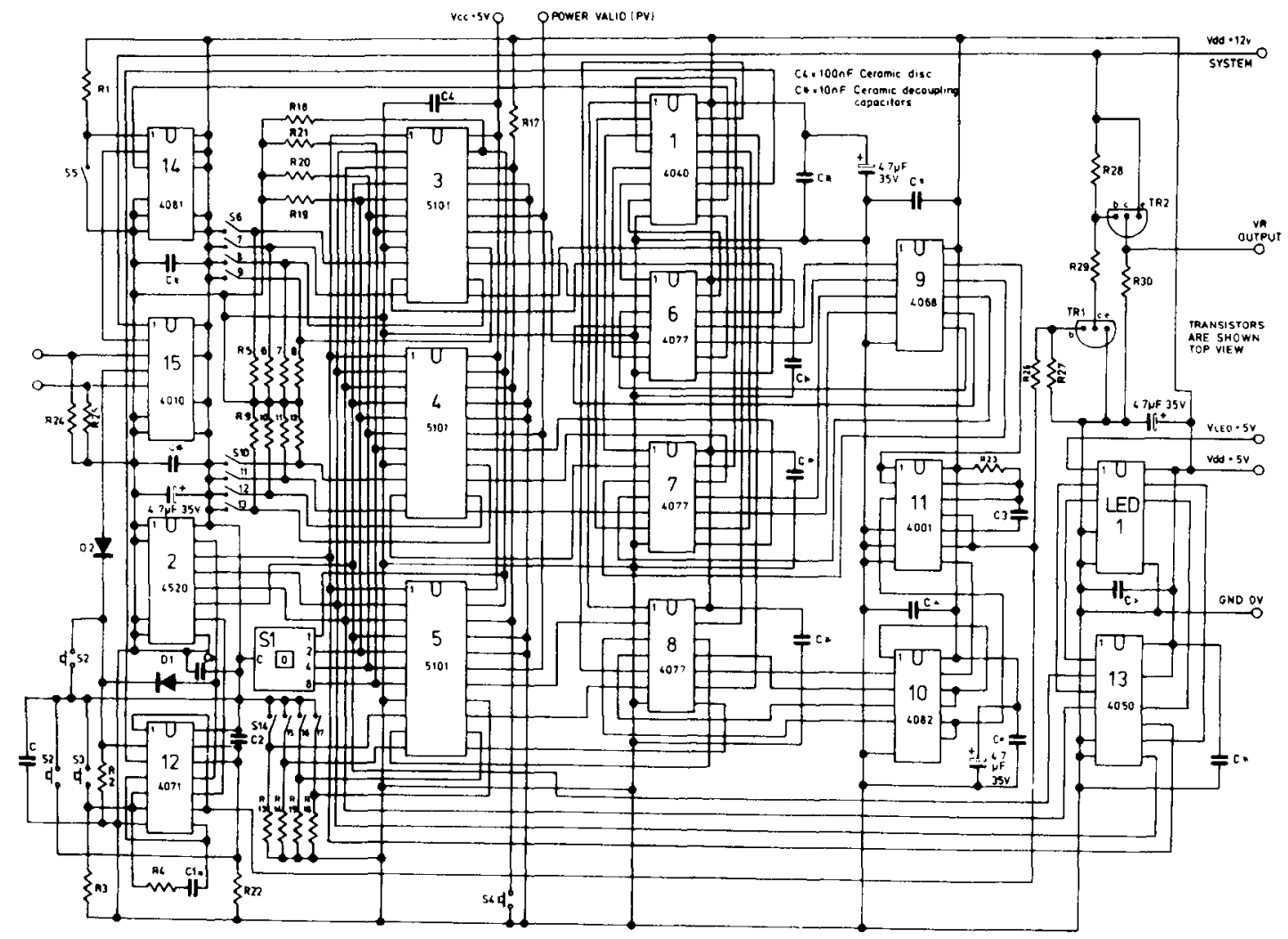

Figure 2. Wiring diagram of VR circuit. 


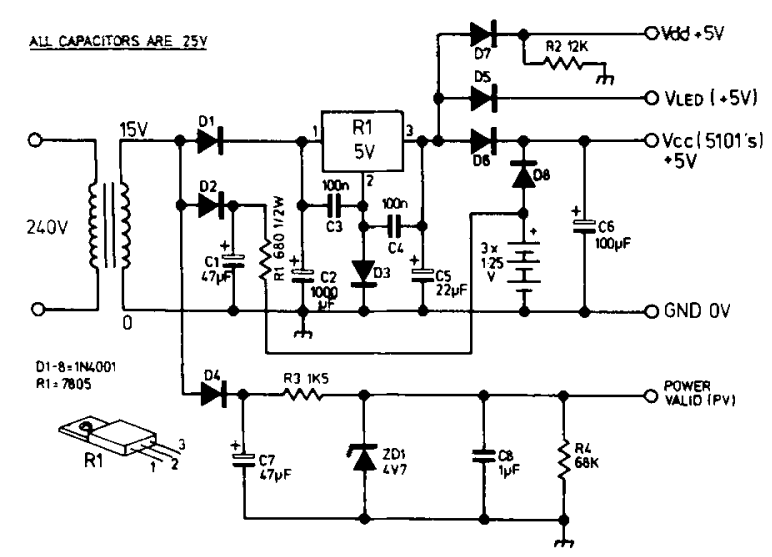

Figure 3. Power supply for VR generator.

power rail, when the main 5-V supply is switched off. This is sufficient to retain in the memories any previously stored sequences. For this facility to operate, the devices require that the chip enable (CE2) input be taken low before the supply falls below $4.75 \mathrm{~V}$, and that it remain low for $\geqslant 650$ nsec following the restoration of the $5-\mathrm{V}$ supply. This is achieved using the components noted in Figure 3. When power is switched off, capacitor C8 discharges through $\mathrm{R} 4$ relatively quickly by comparison with capacitors $\mathrm{C} 2$ and $\mathrm{C} 6$, which maintain the supply to the memories for an adequate period. When power is restored, the Vcc output follows the input waveform rise, whereas the power-valid output is delayed by approximately $15 \mathrm{msec}$ due to the charging of $\mathrm{C} 8$ via $\mathrm{R} 3$.

\section{PROGRAMMING}

Each individual number in the ratio must be stored in memory. This is done using switches S6-S17, which are used to represent the number in binary form. Thus, to program a sequence into memory, it is necessary to specify a sequence number, using S1, and to reset IC 2 using the master clear switch $\mathbf{S 1 8}$. The number is then written (in binary form) using S6-S17, with S6 as the least-significant digit. Operation of the "program" switch, S4, causes the read/write inputs of ICs 3, 4, and 5 to go into the write mode; the word at the data inputs is then written into the specified location. To continue with the sequence, it is necessary to replace the number on switches S6-S17 with the next number in the sequence, after which pressing $\mathrm{S} 3$ increments the address to the next location, and S4 is again used to write the number into the memory. This procedure is continued until the sequence has been completely entered. Resetting is by switches $S 2$ and $S 18 ; S 2$ is a "local reset" that resets ICl to zero; that is, the count of the input pulses is restarted. S18 is a "master reset" that simulates a power-on-clear (POC) signal and resets all counters to zero (although the memories are left unchanged). The POC here is separate from the main system POC.
An inhibit switch, S5, can be used to prevent incoming pulses from reaching $\mathrm{ICl}$, thus preventing any output pulses from occurring. This is achieved by disabling AND gate IC 14a. Such a facility can be of considerable value, especially during the running of an experiment, when for some reason it may be necessary to temporarily "disconnect" the incoming signals from the subject.

Since the INTEL devices are based on a 5-V system, it is necessary to provide a suitable interface between the VR circuit described here and the remainder of the circuits. Figure 4 shows such an interface enabling the $5-\mathrm{V}$-based circuit to be integrated with the 12-V-based circuits previously described. ICs $15 \mathrm{~A}$ and $15 \mathrm{~B}$ act as input buffers, a similar function for the output being provided by TR1 and TR2. A suitable wiring diagram for such a circuit is given in Figure 5 .

\section{ANCILLARY CIRCUITS}

In order to allow maximum benefit to be derived from the various circuits described, a series of ancillary circuits have been devised. It is convenient, for example, to have available a variety of simple logic functions, and several of these can be supplied on a single panel. Four functions have proved particularly useful, AND, OR, flip-flop, and invert. The flip-flops are " $D$ " types with separate set and reset inputs so that latch or toggle modes can be employed, besides the straightforward flip-flop action. Each reset input is connected, via diodes, to the POC circuit so that they are reset when power is applied.

The AND, OR, and invert packages simply have all their inputs and outputs connected to terminal sockets

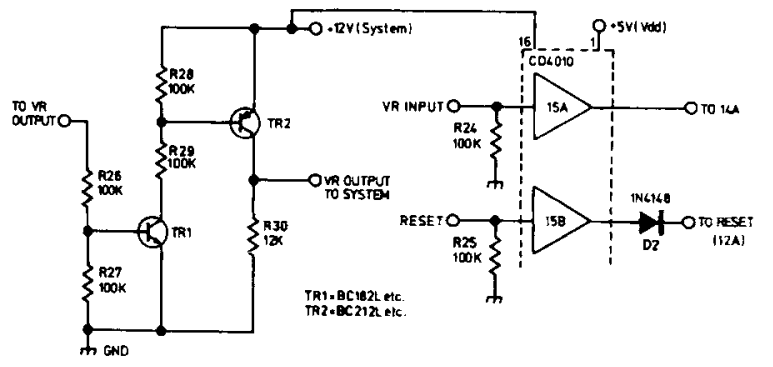

Figure 4. Interface between VR circuit and previous 12-V circuits.

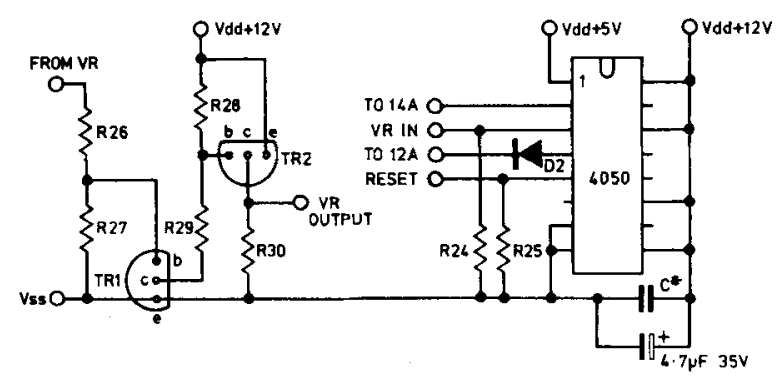

Figure 5. Wiring diagram of VR interface circuit. 
in order that patch leads may be used to wire up a variety of different circuits. Inputs at each of these terminals should be protected against excessive input voltages. Figure 6 gives wiring diagrams for these circuits. A suitable input protection is shown on one input, but should, in fact, be repeated throughout, unless the risk of static charges can be completely eliminated, in which case a single resistor (R2) is all that is necessary to pull the inputs to logical "O." The flip-flops, inverters, AND, and OR functions are supplied by $4013 \mathrm{~s}, 4069 \mathrm{~s}, 4081 \mathrm{~s}$, and $4071 \mathrm{~s}$, respectively. In the prototype equipment, it was convenient to provide terminals on the front panel for logical 1 and 0 connections; these simply were connections to VDD via a resistor and to ground (VSS).

Figure 7 shows the circuit and wiring diagrams for the POC function, used with all circuits so far described to provide a reset when equipment is first switched on. The circuit operates by using the outputs of six inverters of a 4069 (connected together to increase the drive capability) as a buffer: At the instant the equipment is switched on, capacitor $\mathrm{Cl}$ has no voltage across it, and hence, the outputs of the inverters are at logical 1 . Since $\mathrm{Cl}$ is connected to the CMOS power supply via $\mathrm{R} 1$, however, it rapidly charges, taking the inputs to the inverters high after approximately $100 \mathrm{msec}$ and hence causing the outputs to switch to the stable state of logical 0. A master reset switch, S1, is provided to permit discharging of $\mathrm{Cl}$ and hence to reset all circuits together.

Finally, it is appropriate to consider three remaining circuits that, while not themselves concerned with schedule generation, have proved remarkably useful in operating such equipment. These consist of a simple logic probe for CMOS, capable of detecting both logical 1 states and transient pulses, an elapsed-time meter, and a test pulse circuit capable of providing a series of input pulses and hence simulating an experimental subject. The latter circuit is of considerable value in setting up and testing experimental equipment.

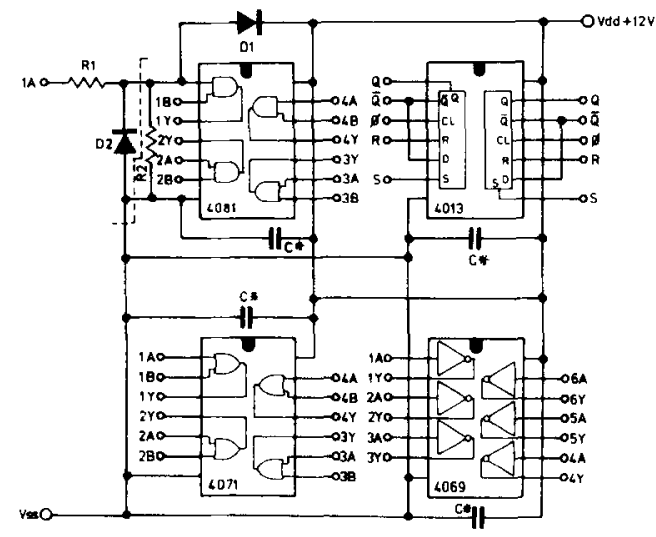

Figure 6. Connection diagram of ancillary circuits showing input protection on one input: $R 1=1 \mathrm{~K}, \mathrm{R} 2=100 \mathrm{~K}, \mathrm{D} 1-\mathrm{D} 2=$ IN4001.
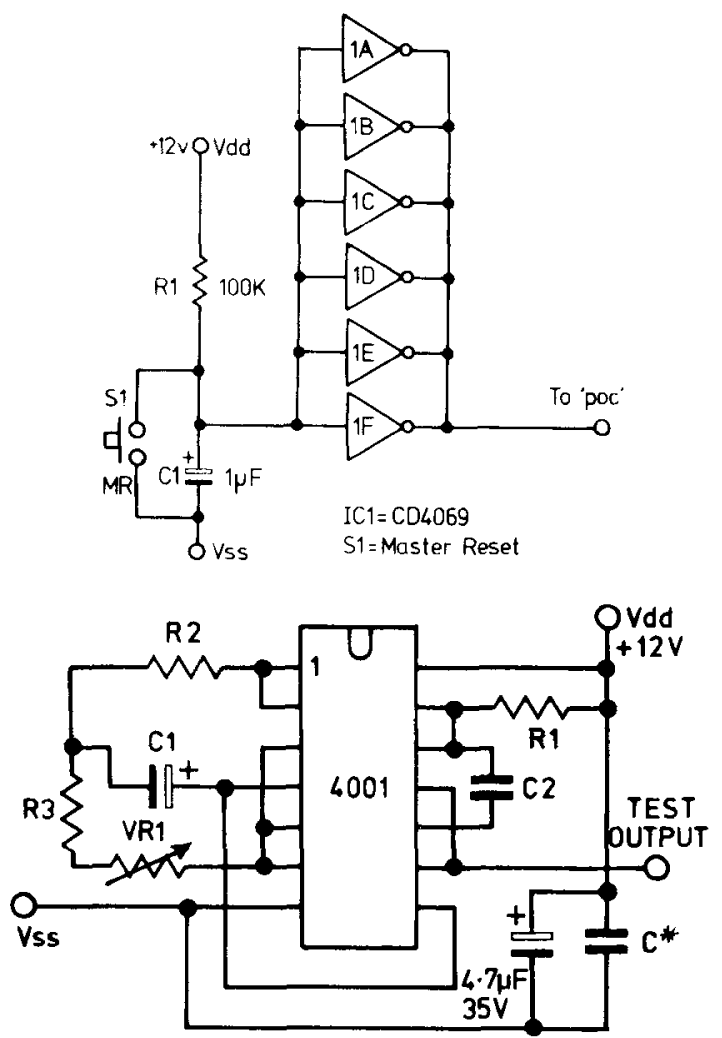

Figure 7. Structure and wiring diagrams of power-on-clear circuit.

Figure 8 shows the circuit for the elapsed-time meter, built around the AY-5-1224A clock chip, connected via display drivers (TR1-4 and R11-21) to four sevensegment LED displays (MAN3M or similar). The time base for the chip is provided by the oscillator formed by NAND gates IC $2 a$ and IC $2 b$ set, with VR 1, to oscillate at $50 \mathrm{~Hz}$. This signal is switched on or off by gating with the flip-flop formed by IC $2 \mathrm{c}$ and IC $2 \mathrm{~d}$, which provides the start/stop functions. The chip is "programmed" using diodes between the segment outputs and the clock strobe line. D2 sets the chip in 24-h mode, and D3 holds the clock in a "set minutes" condition that causes the clock to count in minutes and seconds instead of hours and minutes (the "set minutes" advances the display at a $1-\mathrm{Hz}$ rate).

Reset to zero is achieved by connecting segment $C$ to strobe with the reset button or via TR6. TR6 is driven by the monostable formed by ICs $3 a$ and $3 b$, which when triggered, supplies a $15-\mathrm{msec}$ pulse to the base of TR6 resetting the clock. Triggering of the monostable is caused by a pulse at the remote reset input circuit formed by TR9 and IC 3e. Similar input pulses buffered by TR7 and TR 8 can be used to remotely operate the start/stop functions by driving the IC $2 \mathrm{c} / \mathrm{IC} 2 \mathrm{~d}$ flip-flop.

For greater flexibility, the prototype meter is battery operated. In order to conserve battery life, the display is normally off, giving a quiescent current of approximately $2.5 \mathrm{~mA}$. IC $3 \mathrm{c}$ and IC $3 \mathrm{~d}$ are connected to form 

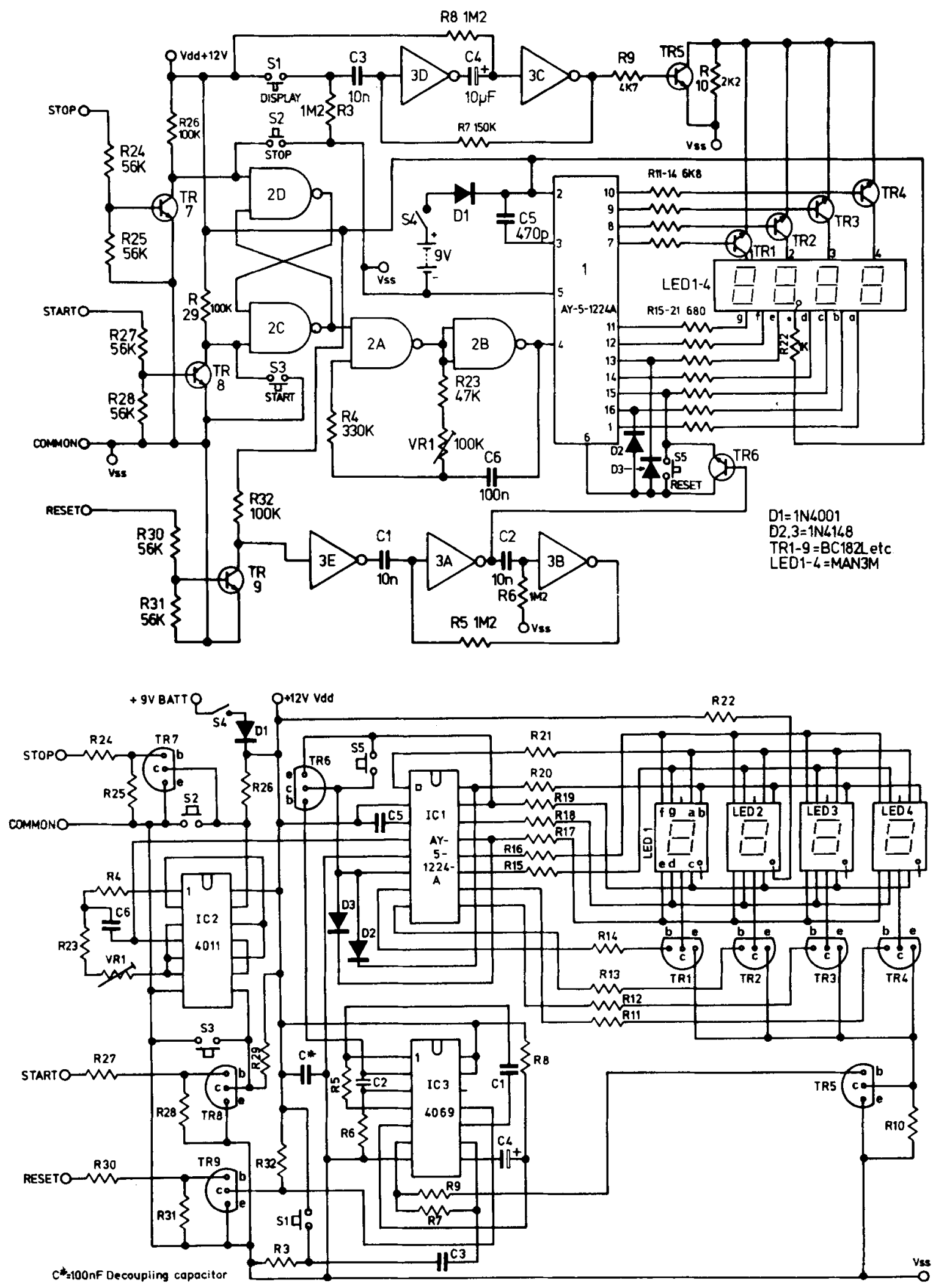

Figure 8. Logical circuit and wiring diagrams for elapsed-time meter. 

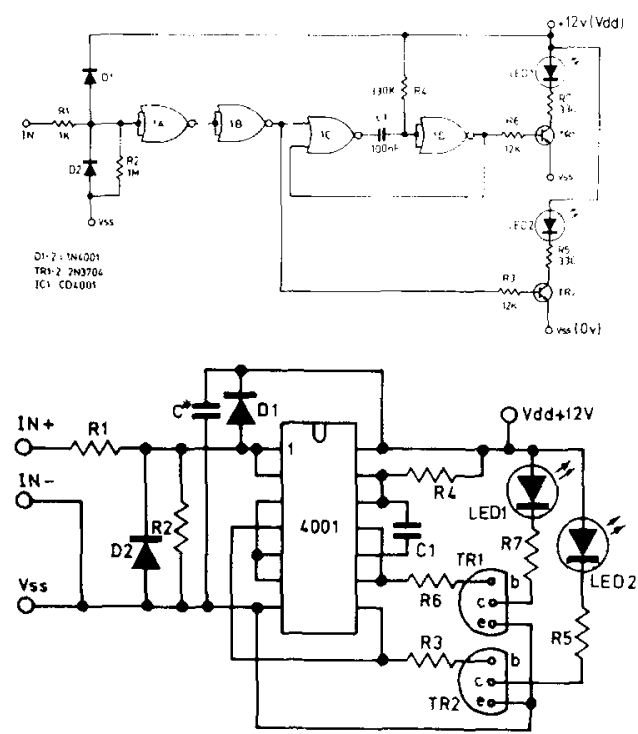

Figure 9. Circuit and wiring diagrams of logic probe.

a monostable with a period of $15 \mathrm{sec}$. This can be used to switch on the display by turning on TR5, thus shorting out R10, which provides a ground reference for the chip. Full current is enabled to the display for the duration of the monostable period, after which, the display is again turned off without interrupting the normal functions of the chip. Triggering of the monostable is caused by closure of pushbutton switch S1. In order to provide a divider between the minutes and seconds, the decimal point of the second MAN3M is illuminated by means of $R 22$.

Figure 9 provides a suitable circuit for a logic probe, giving an illuminated indication of either a transient pulse or a logical 1 state. Resistor R1, with diodes D1 and D2, prevents excessive voltages from being applied to the input of IC 1a, which is pulled low by $\mathrm{R} 2$. When the input is taken high, it causes the output of $\mathrm{IC} \mathrm{lb}$ to go high and turns on TR2, causing LED 2 to conduct and indicate a logical 1 at the input. In order to provide an indication of very short pulses, the output of IC $1 \mathrm{~b}$ is also used to trigger a monostable formed by IC $1 \mathrm{c}$ and IC $1 \mathrm{~d}$. This gives a pulse length of approximately $50 \mathrm{msec}$, which turns on LED 1 via TR1, enabling shortduration pulses to be clearly visible. Like the elapsedtime meter, the probe can be battery operated, although a more conveneint arrangement might be to incorporate the probe into, for example, the logic panel, providing an input socket to which any point in the circuitry may be attached using a suitable lead.

To help in testing experimental circuits, the circuit shown in Figure 10 was devised to provide input pulses for other circuits and hence to simulate an experimental subject. This consists of an oscillator formed by IC 1 a and IC $1 \mathrm{~b}$ and a monostable formed by IC $1 \mathrm{c}$ and IC $1 \mathrm{~d}$. The monostable, when triggered, produces pulses similar to those used as inputs from experimental equipment (microswitch closure, etc.). The frequency of the oscillator, and hence the frequency with which the monostable is triggered, is adjusted using VR 1 . In the circuit shown, the range of frequencies is from approximately $.6 \mathrm{~Hz}$ to $3 \mathrm{~Hz}$, but this may be changed by altering the value of $\mathrm{Cl}$ according to the formula $\mathrm{t} \bumpeq 1.4 \mathrm{Cl} \times(\mathrm{VR} 1+\mathrm{R} 3$ ) sec, where $\mathrm{C}$ is in microfarads and VR $1+R 3$ is in megohms. The practical limit on the maximum value of $\mathrm{Cl}$ is $47 \mathrm{microF}, 16 \mathrm{~V}$ (tantalum). If longer time periods are required, VR 1 may be increased to 2 megohms.

Wiring diagrams show power supply decoupling capacitors marked $\mathrm{C}^{*}$. These prevent the interaction of various circuit elements and should be between 10 nanoF and 100 nanoF $25 . \mathrm{V}$ disk ceramic. They are not shown on the circuit diagrams for clarity. Also included on the wiring diagrams are tantalum capacitors to provide low-frequency decoupling. It is good practice to place one across the supply every five IC packages. All components are drawn top view.

\section{CONCLUSIONS}

Although the equipment described in this paper is relatively simple in principle, the various circuits described can be interconnected to provide remarkably complex circuits. By appropriate use of the logic panel described above, it has proved possible to combine circuits giving, for example, a variety of chain and tandem schedules, second order schedules, concurrent schedules, and so forth. Indeed, the equipment has so far proved capable of providing all the circuits that have been required since the system was built. Specific designs for chain, tandem, conjoint, and concurrent schedules are available from the second author on request. It is expected, however, that the simplicity of the equipment will make design of such complex circuits relatively easy.
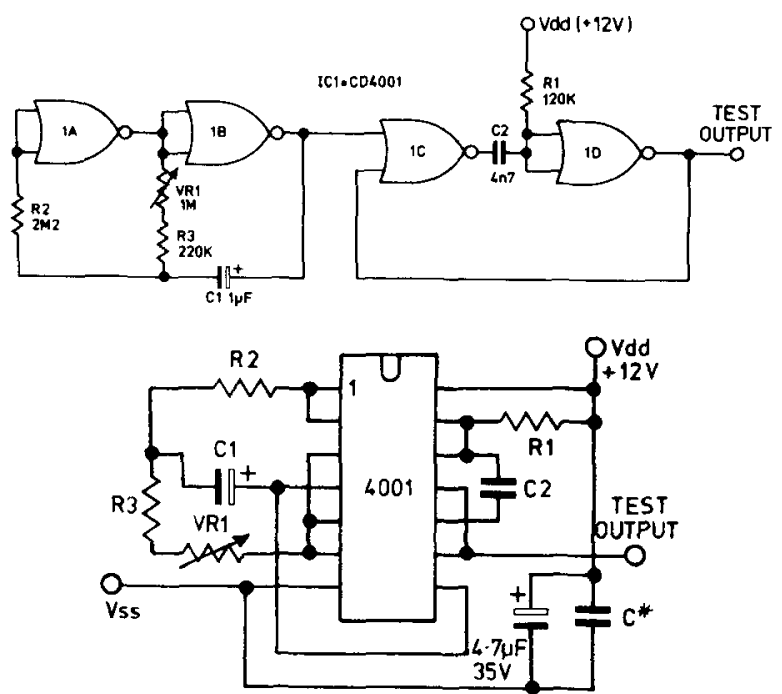

Figure 10. Circuit and wiring diagrams of test pulse generator. 


\section{REFERENCES}

Aylesbury, L. J., \& Owens, R. G. Low-cost equipment for schedule generation I: Fixed and random ratio schedules. Behavior Research Methods \& Instrumentation, 1977, 9, 247 252.

Ferster, C. B., \& Skinner, B. F. Schedules of reinforcement. New York: Appleton-Century-Crofts, 1957.
Owens, R. G., \& Aylesbury, L. J. Low-cost equipment for schedule generation II: Time dependent schedules. Behavior Research Methods \& Instrumentation, 1978, 10, 29-32.

(Received for publication November 14, 1978; accepted December 28, 1978.) 